\title{
A PROPOSAL FOR IMPROVE THE LIFE- TIME OF WIRELESS SENSOR NETWORK
}

\author{
Tran Cong Hung1 and Nguyen Hong Quan2 \\ 1Post \& Telecommunications Institute of Technology, Vietnam \\ 2University of Science, Ho Chi Minh City, Vietnam
}

\begin{abstract}
In the Wireless Sensor Network (WSN), sensor nodes are connected together through radio frequency $(R F)$. Routing protocol is used to transmit data among sensor nodes. In the paper, we proposed a new routing protocol based on LEACH protocol. This is energy-efficient clustering algorithm. The proposed protocol enlarges WSN life-time by considering remaining energy and distance from nodes to BS in the election of cluster head. Comparing the result simulation between LEACH and proposed protocol showed that proposed protocol will prolong the network life-time.
\end{abstract}

\section{KEYWORD}

LEACH, Energy efficient, WSN.

\section{INTRODUCTION}

The development of sensor technology allows the procedure sensor to produce large quantities and reasonable price. Sensors are in charge of sensing the environment for the specific application. The sensor nodes are connected together via radio communication that are called wireless sensor network. Wireless sensor networks have been applied in military and civilian fields. WSN is classified as ad-hoc network, however the routing protocols used in WSN having many differences to traditional ad-hoc networks in which the most important issue is network energy consumption. the routing protocols used in WSN focus on how to increase the life-time of the entire network rather than looking for the shortest route, reducing time-delay or optimizing bandwidth as in the traditional network protocol such as mobile ad-hoc networks or cellular networks. In addition, the deployment of a large number of sensor nodes makes topology construction basing on IP protocols difficult to deploy in WSN.

Because there are many differences, there are many of WSN routing algorithms proposed to solve this problem. The routing methods depend on the characteristics of sensor nodes, application requirements and deployed environment of WSN.

In this paper, we propose a new routing protocol basing on LEACH protocol for wireless sensor networks to extend the lifetime of wireless sensor networks in terms of BS placed in the sensor area.

The remainder of the paper is organized as follows. In Section 2, we briefly review related work. Section 3 describes the proposed protocol. Section 4 presents the detail of result simulation and compares with LEACH protocol. Finally, Section 5 gives conclusion and future work. 


\section{RELATED WORK}

Hierarchical routing protocol or cluster-based routing, they will divide WSN into smaller area sections called clusters, this division enlarge network scalability and uses energy efficiently in entire network.

The main idea of the hierarchical routing protocols is that the nodes only communicate with their Cluster Head $(\mathrm{CH})$, each cluster has a Cluster Head. $\mathrm{CHs}$ are responsible for receiving data from the nodes in clusters, synthesizing data before transferring their data to base station (BS).

Low-Energy Adaptive Clustering Hierarchy (LEACH) [1] is the first protocol in the cluster routing, a lot of clustering protocols basing on them. LEACH will divide action into many rounds. Each round consists two main phases: clustering phase and stable phase. There are two small phases in the clustering phase: election clusters head and the clustering. The election of the LEACH cluster Head is random. At the beginning of each round, the nodes will generate a random number between 0 and 1 , the random numbers will compare with the threshold $T(n)$. If that number is less than $\mathrm{T}(\mathrm{n})$, that nodes will be $\mathrm{CH}$ in the current round.

$$
\mathrm{T}(\mathrm{n})=\left\{\begin{array}{l}
\frac{p}{1-p *\left(r \mathrm{mod} \frac{1}{p}\right)} \\
0
\end{array} \text { if } \mathrm{n} \in \mathrm{G}\right.
$$

After electing $\mathrm{CH}$, the $\mathrm{CHs}$ will broadcast messages into the entire network, the nodes base on the received signal strength indicator (RSSI) to decide to join their $\mathrm{CH}$, and this is the principle of the clusters in LEACH.

After the clustering phase is stable phase. In this phase, the nodes in the same cluster will transfer the data to $\mathrm{CH}$, the $\mathrm{CH}$ will create TDMA schedule and broadcast to the nodes in the cluster, TDMA schedule are created by the cluster head which will define time to transfer data for nodes, the normal nodes base on TDMA schedule knowing their transmission time. At that time, this nodes will transfer the data to its $\mathrm{CH}$, if the normal nodes are not in transfering time, they will be on sleep mode to save energy. After receiving data from the normal node in cluster, the $\mathrm{CHs}$ synthesize all the data and send the aggregated data to the BS through a single hop transmit methods.

This cluster will reduce the data transmitted directly to the BS. In addition, the use of Time Division Multiple Access (TDMA) frame for data transmission from the node to the $\mathrm{CH}$ will avoid a collision between the nodes in the cluster to all data.

However, random election also has many limitations, including the biggest drawbacks is the random vote will make the high-energy nodes have the same probability of becoming cluster all nodes have the same power low volume.

The $\mathrm{CHs}$ functions have sensing such normal nodes in addition, receive data from the nodes in the cluster, aggregate and transmit data to the BS data so all clusters consumes more energy than nodes generally, if a low-energy node becomes cluster will lead to all these buttons will consume more energy, while the low-energy node will quickly stop working load imbalance of the whole network. 
However, random election also has many limitations. The biggest drawbacks is the random election making the high-energy nodes have the same probability of becoming cluster as lowenergy nodes.

The CHs functions have sensing as normal nodes. In addition, $\mathrm{CHs}$ also receive data from the nodes in the cluster, aggregate data and transmit data to the $\mathrm{BS}$, so that $\mathrm{CH}$ consumes more energy than normal node. If low-energy nodes becomes $\mathrm{CH}$, it will consume more energy at the result, the low-energy nodes will quickly stop working.

Moreover, single-hop transition methods used in LEACH make $\mathrm{CHs}$ in a far distance from BS consume more energy than $\mathrm{CH}$ s close to $\mathrm{BS}$ so that $\mathrm{CHs}$ far from $\mathrm{BS}$ die quickly.

To solve this problem, many protocols have been proposed which have two main ways: optimizing the election CHs by considering nodes energy issues in the optimal threshold $\mathrm{T}(\mathrm{n})$ and transmitting data to the BS through multi-hop communication methods.

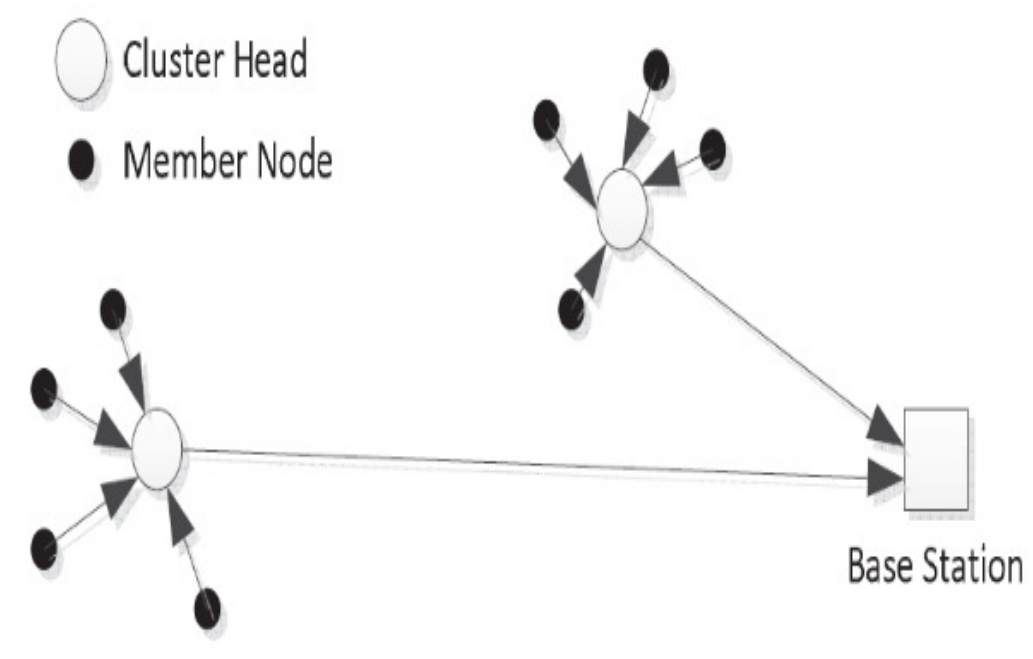

Figure 1. Transmit data in LEACH.

The multi-hop communication method proposed in M-LEACH protocol (multi-hop LEACH) protocol is the most popular protocol. The main idea of this protocol is that the farther CHs will transmit their data to the $\mathrm{CHs}$ which are closer to BS. The near $\mathrm{CHs}$ are in task of forwarding far CHs' data to BS.

Multi-hops communication protocol [2] has been proven to consume less energy than single-hop communication in large networks. 


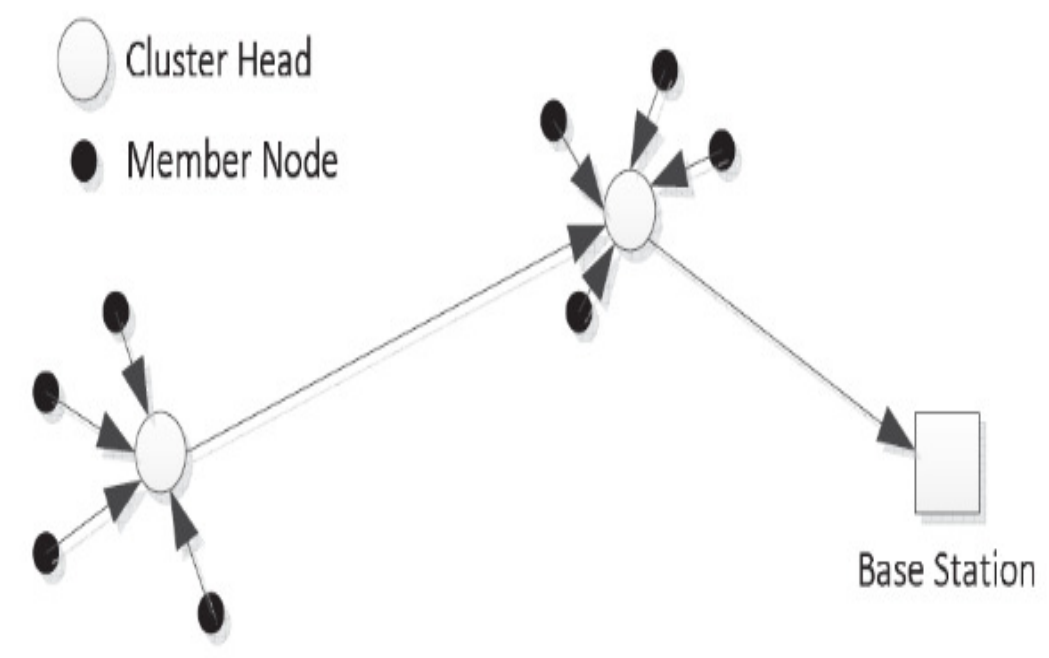

Figure 2. Transmit data in Multi-hop LEACH.

Other way to enlarge the lifetime network based on clustering algorithm is that nodes' energy considering, in which the most considered coefficients are CHs election based on the nodes' energy remain. DCHS protocol [3] is developed basing on LEACH algorithm, it elects CHs based on nodes' energy. At that time, threshold $\mathrm{T}(\mathrm{n})_{\text {new }}$ are defined:

$$
T(n)_{\text {new }}=\frac{p}{1-p[r \bmod (1 / p)]} \times \frac{E_{n_{-} \text {carrent }}}{E_{n_{-} \max }}
$$

Where: $\mathrm{E}_{\mathrm{n}_{-} \text {current }}$ is remaining energy of nodes; $\mathrm{E}_{\mathrm{n} \_ \text {max }}$ is initial energy of the node.

The election of CHs bases on nodes' remaining energy which is more efficient. The probability of high-energy nodes become $\mathrm{CHs}$ is higher than the low-energy node.

In addition, all of the elected Cluster Head, DCHS and LEACH does not consider the distance problem, the protocol proposed in the paper method will increase the lifetime of the network based on the selection of clusters based on all the remaining energy and spacing of the buttons is described in the next section.

\section{THE PROPOSED PROTOCOL}

As same as LEACH protocol, the proposed protocol operates into rounds. Each round includes two phases: clustering phase and data transmission phase. In clustering phase, the $\mathrm{CH}$ election is based on two factors: the remaining energy of nodes and the distance from the node to the BS.

At the beginning of each round, the sensor nodes will generate a random number in the range $(0$, 1 ), this value is compared with the threshold $T(n)$. Before calculating the threshold $T(n)$ we define d (i) $=\sqrt{\left(X_{i}-X_{B S}\right)^{2}+\left(Y_{i}-Y_{B S}\right)^{2}}$.

Where $\mathrm{d}$ (i) is the distance from node $\mathrm{i}$ to $\mathrm{BS} ; \mathrm{n}$ is the total number of nodes in sensor networks; $(\mathrm{Xi}, \mathrm{Yi})$ are the coordinates i node in the network; $\left(\mathrm{X}_{\mathrm{BS}}, \mathrm{Y}_{\mathrm{BS}}\right)$ is of $\mathrm{BS}$ coordinates; $\mathrm{E}$ (i) is the 
residual energy of the nodes in the round $\mathrm{r}$; then the threshold $\mathrm{T}(\mathrm{n})$ will be calculated according to the expression:

$$
\mathrm{T}(\mathrm{n})=\left\{\begin{array}{l}
\frac{p}{1-p *\left(r \bmod \frac{1}{p}\right)} \\
\text { Otherwise }
\end{array} \frac{E(\mathrm{i})}{d(\mathrm{i})} \quad \text { if } \mathrm{n} \in \mathrm{G}\right.
$$

With $\mathrm{G}$ is the set of nodes which have not become $\mathrm{CH}$ in the previous round; $\mathrm{p}$ is desired percentage per the total number which want to be $\mathrm{CHs}$. As normal, $\mathrm{p}$ in the range of $0.05-0.1 ; \mathrm{r}$ is the current round.

If the random value of the node is smaller than threshold $\mathrm{T}$ (n), it becomes $\mathrm{CH}$ in the next round. The using of remaining energy parameters of nodes will increase the probability of becoming $\mathrm{CHs}$ of high-energy nodes, corresponding to basing on the nodes' distance to BS to make the near nodes from BS have higher probability to become $\mathrm{CHs}$ than nodes far nodes from BS.

After the election of $\mathrm{CH}$, the $\mathrm{CHs}$ sends their location information to the $\mathrm{BS}$, and it will broadcast the INVITE message to entire network. This message has the same signal strength. The normal nodes receive this message and then if they want to join this cluster, they will reply ACCEPT message. If a node receives multiple INVITE messages, it depends on the signal strength of the INVITE message, after that it will decide to join in higher signal strong cluster.

If the $\mathrm{CH}$ does not receive any ACCEPT messages, it will resend the INVITE message again. If it still not receive ACCEPT message, it will send data to BS directly without creating TDMA schedule.

After the clustering phase, nodes are transmit their data, the transmission of data via two main steps, transmit data from nodes to $\mathrm{CHs}$ and transmission data from the $\mathrm{CHs}$ to the BS. Data during transmission from the node to the $\mathrm{CH}, \mathrm{CH}$ creates a TDMA table specified timeframe for the data transmission node in the cluster then it will promote the TDMA table to the nodes in its cluster, the nodes in the cluster based on this table will know it is time to send data to $\mathrm{CH}$, in the time remaining idle nodes to save energy, the method uses TDMA transmission allows nodes to the cluster data avoid collisions with all other nodes

\section{SIMULATION}

In this section, we used matlab to simulate our proposed protocol. Assuming that there are 100 nodes randomly deployed in a region of size $100 \mathrm{mx} 100 \mathrm{~m}$. BS has coordinates $(50,50)$ are deployed in the sensor area. Nodes are fixed, no mobile and determined coordinates. Initial energy of the nodes is $0.25 \mathrm{~J}$ and $0.5 \mathrm{~J}$, data packet size is $4000 \mathrm{bits}$. Probability become clusters head of network 0.05 or $5 \%$.

We will use energy model in [1]. 
International Journal of Computer Networks \& Communications (IJCNC) Vol.6, No.5, September 2014

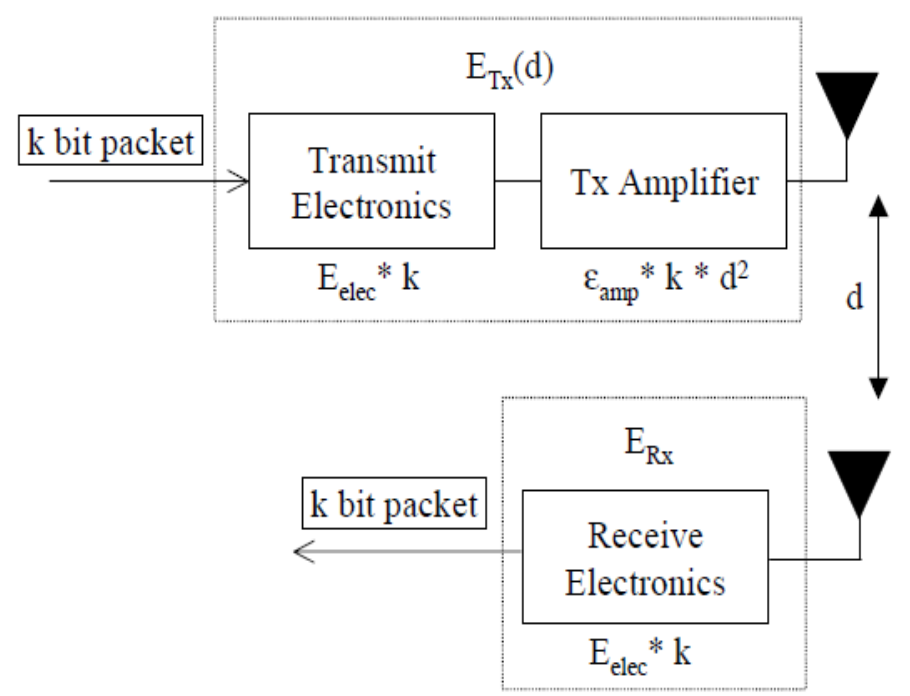

Figure 3. Energy model used in LEACH protocol

Parameter used in simulation:

\begin{tabular}{|l|l|}
\hline Decription & Parameter \\
\hline Network size & $100 \mathrm{~m}^{*} 100 \mathrm{~m}$ \\
\hline Total node $(\mathrm{n})$ & 100 \\
\hline CH's percentage $(\mathrm{p})$ & 0.05 \\
\hline Coordiner BS $\left(\mathrm{X}_{\mathrm{BS}}, \mathrm{Y}_{\mathrm{BS}}\right)$ & $(50,50)$ \\
\hline Data Packet $(\mathrm{k})$ & 4000 \\
\hline Energy Init $\left(\mathrm{E}_{\mathrm{O}}\right)$ & $0.25, \quad 0.5,1 \mathrm{~J}$ \\
\hline$\left(\mathrm{E}_{\mathrm{elec}}\right)$ & $50 \mathrm{~nJ} / \mathrm{bit}$ \\
\hline $\mathrm{E}_{\mathrm{mp}}$ & $0.0013 \mathrm{pJ} / \mathrm{bit} / \mathrm{m} 4$ \\
\hline $\mathrm{E}_{\mathrm{fs}}$ & $10 \mathrm{pJ} / \mathrm{bit} / \mathrm{m} 2$ \\
\hline do & $\sqrt{\frac{E f s}{E m p}}=87 \mathrm{~m}$ \\
\hline
\end{tabular}

Based on the energy model used in [1], we have:

$$
E_{T X}=\left\{\begin{array}{l}
k E_{\text {elec }}+k E_{f s} d^{2}, d<d o \\
k E_{\text {elec }}+k E_{m p} d^{4}, d>=d o
\end{array}\right.
$$


International Journal of Computer Networks \& Communications (IJCNC) Vol.6, No.5, September 2014

Where $\mathrm{k}$ is the number of transmitted bits, $\mathrm{E}_{\mathrm{elec}}$ is the energy consumption to run transmiter or $\begin{array}{llllll}\text { receiver. } & \mathrm{E}_{\mathrm{mp}} & \text { or } & \mathrm{E}_{\mathrm{fs}} & \text { depend } & \text { on } \\ \end{array}$ To receive $\mathrm{k}$ bits of data of energy consumption $\mathrm{E}_{\mathrm{RX}}$ is:

$$
E_{R X}=k^{*} E_{\text {elec }}
$$

Result for proposed protocol:

To simulate the effection of routing protocol in WSN, we use [4]:

- Stability period: It is duration of network operation from start till first node dies

- Network lifetime: Network lifetime is duration from start till last node is alive

- Instability period: It is duration of network operation from first node dies till the least node dies

- Packet to BS: It is rate of successful data delivery to BS from $\mathrm{CHs}$

Table 1. Comparision LEACH protocol and Proposed protocol with Eo $=0.25 \mathrm{~J}$

\begin{tabular}{|l|l|l|l|}
\hline Protocol & Stability period & Network lifetime & Instability period \\
\hline LEACH & 445 & 700 & 255 \\
\hline $\begin{array}{l}\text { Proposed } \\
\text { protocol }\end{array}$ & 690 & 1229 & 539 \\
\hline
\end{tabular}

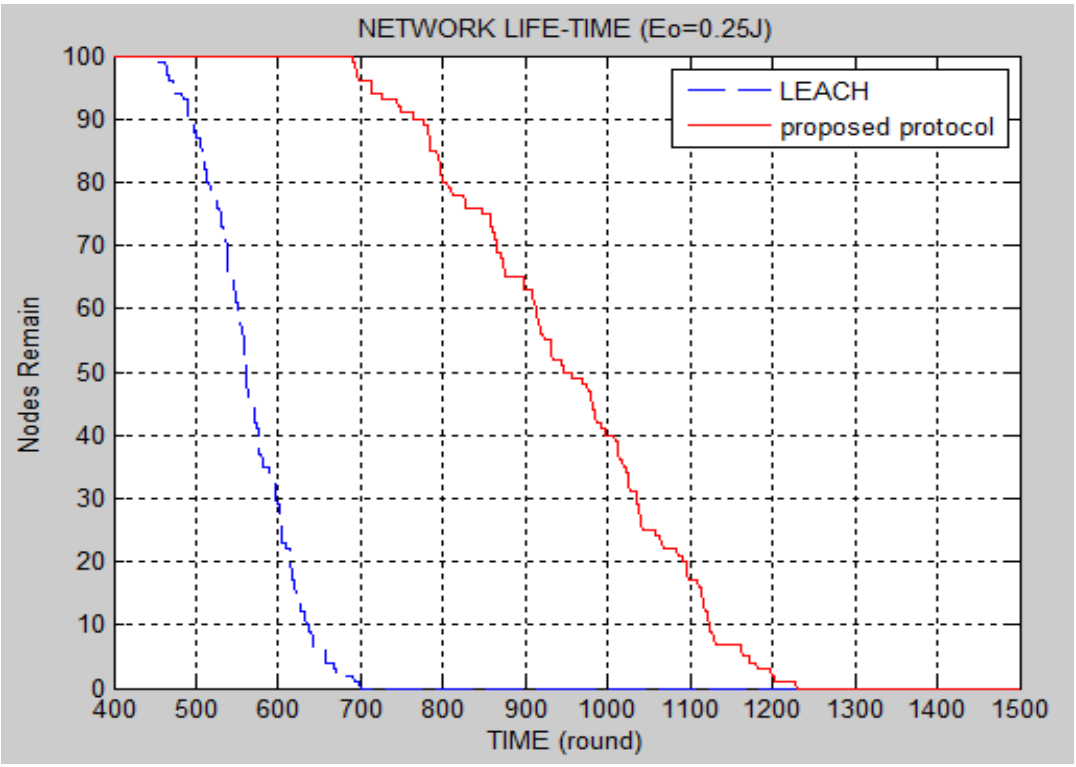

Figure 4. Compare network life-time between LEACH and Proposed Protocol $(\mathrm{Eo}=0.25 \mathrm{~J})$ 
International Journal of Computer Networks \& Communications (IJCNC) Vol.6, No.5, September 2014

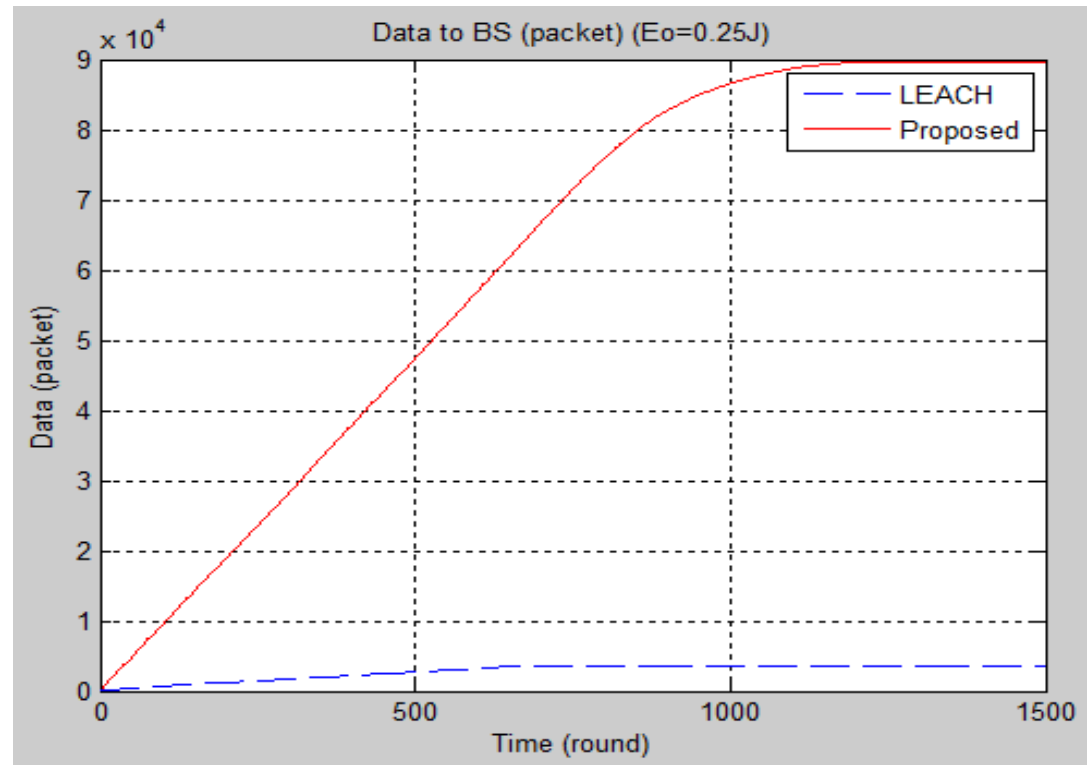

Figure 5. Data to BS between LEACH and proposed protocol $(\mathrm{Eo}=0.25 \mathrm{~J})$

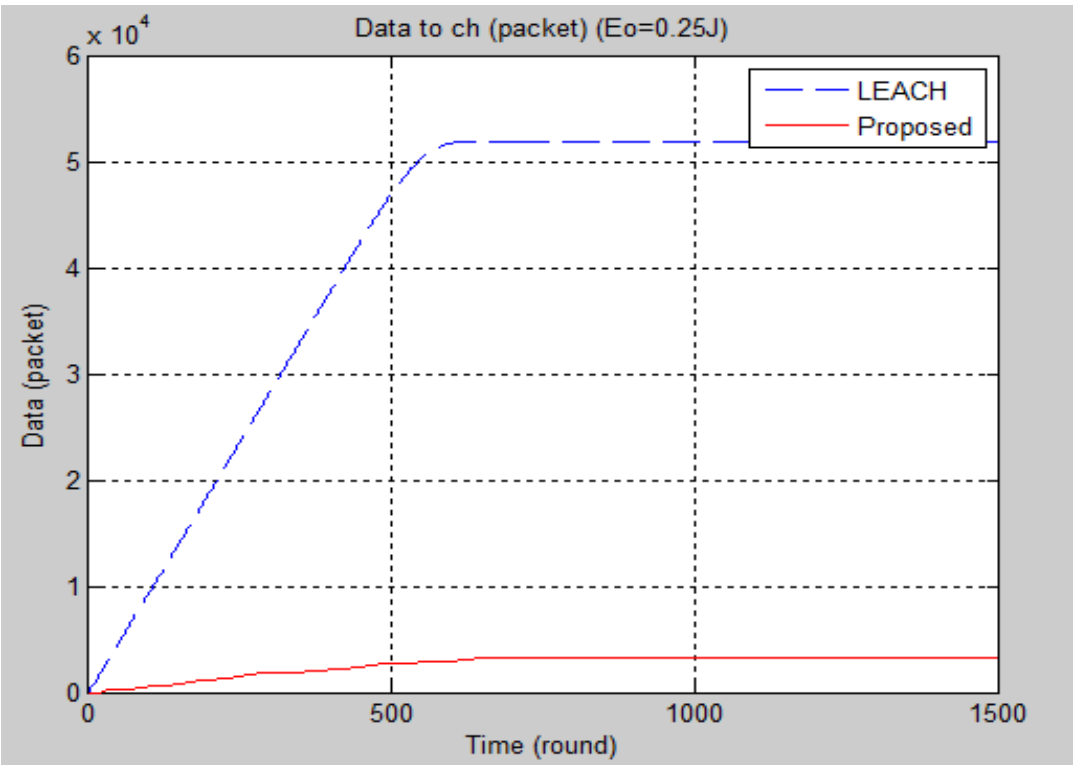

Figure 6. Data to $\mathrm{CH}$ between $\mathrm{LEACH}$ and proposed protocol $(\mathrm{Eo}=0.25 \mathrm{~J})$

Table 2. Comparision LEACH protocol and Proposed protocol with Eo $=0.5 \mathrm{~J}$

\begin{tabular}{|l|l|l|l|}
\hline Protocol & Stability period & Network lifetime & Instability period \\
\hline LEACH & 861 & 1289 & 428 \\
\hline $\begin{array}{l}\text { Proposed } \\
\text { protocol }\end{array}$ & 1126 & 2294 & 1168 \\
\hline
\end{tabular}


International Journal of Computer Networks \& Communications (IJCNC) Vol.6, No.5, September 2014

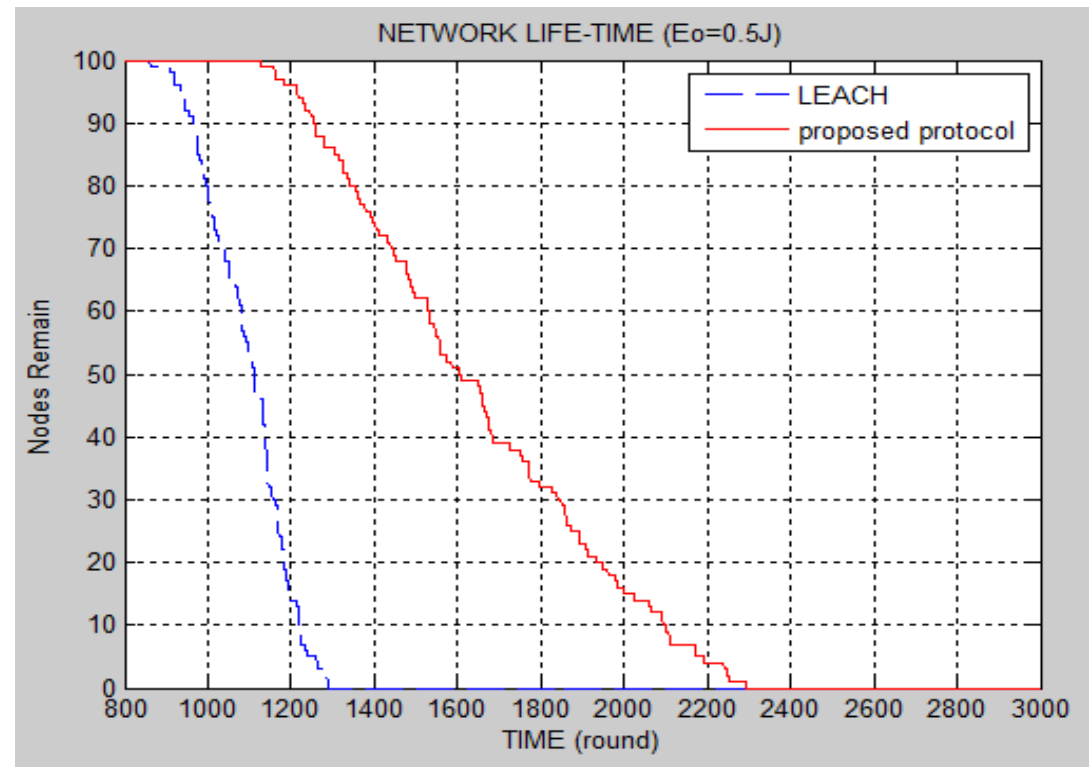

Figure 7. Compare network life-time between LEACH and Proposed protocol $($ Eo $=0.5 \mathrm{~J})$

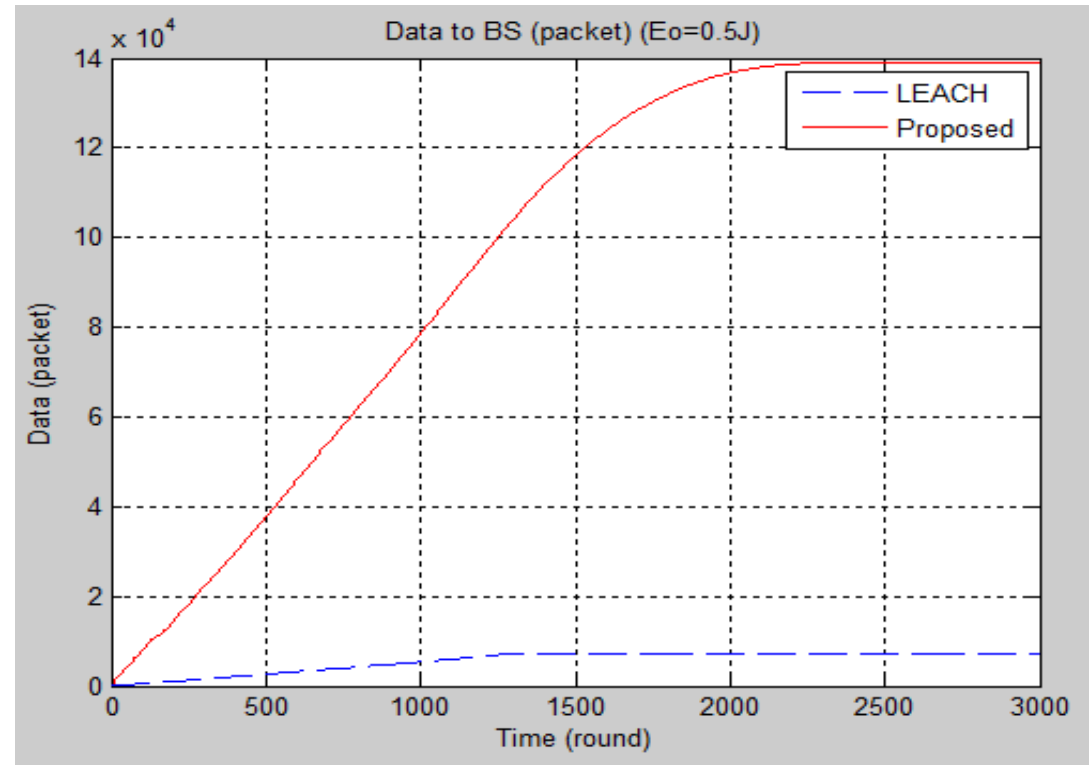

Figure 8. Data to BS between LEACH and proposed protocol $(\mathrm{Eo}=0.5 \mathrm{~J})$ 
International Journal of Computer Networks \& Communications (IJCNC) Vol.6, No.5, September 2014

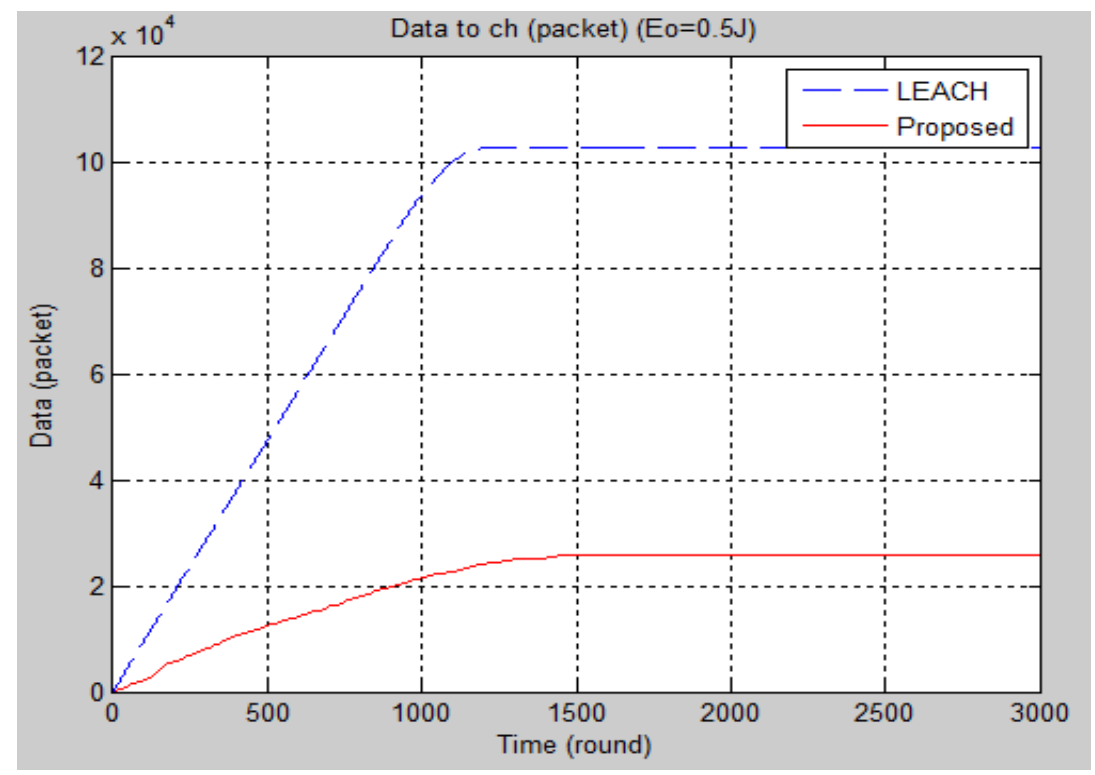

Figure 9. Data to $\mathrm{CH}$ between $\mathrm{LEACH}$ and proposed protocol $(\mathrm{Eo}=0.5 \mathrm{~J})$

The result of simutaion show that, proposed protocol increed network life-time up 75\% compared with LEACH protocol.

To evaluate the effect of the proposed protocol, we use some following parameter to measure simulation results. Those are 10\% Node Dead (TND), Haft Node Dead (HND) and Full Nodes Dead (FND).

Table 3. Compare TND, HND and FND between LEACH and Proposed protocol with Eo $=0.25 \mathrm{~J}$

\begin{tabular}{|l|l|l|l|}
\hline Parameter & $\begin{array}{l}10 \% \\
\text { dead }\end{array}$ & $\begin{array}{l}50 \% \\
\text { dead }\end{array}$ & $\begin{array}{l}100 \% \\
\text { Dead }\end{array}$ \\
\hline LEACH & 492 & 561 & 700 \\
\hline $\begin{array}{l}\text { Proposed } \\
\text { protocol }\end{array}$ & 764 & 946 & 1229 \\
\hline
\end{tabular}

According to the simulation results, the initial energy is $0.25 \mathrm{~J}$. In compare with LEACH protocol, proposed protocol increases TDN by 55\%, HND by $68 \%$ and FDN by $75 \%$.

Table 4. Compare TND, HND and FND between LEACH and Proposed protocol with Eo $=0.5 \mathrm{~J}$

\begin{tabular}{|l|l|l|l|}
\hline Parameter & $\begin{array}{l}10 \% \\
\text { dead }\end{array}$ & $\begin{array}{l}50 \% \\
\text { dead }\end{array}$ & $\begin{array}{l}100 \% \\
\text { Dead }\end{array}$ \\
\hline LEACH & 965 & 1111 & 1289 \\
\hline $\begin{array}{l}\text { Proposed } \\
\text { protocol }\end{array}$ & 1254 & 1603 & 2294 \\
\hline
\end{tabular}


With initial energy Eo $=0.5 \mathrm{~J}$, the proposed protocol can make network lifetime increase, respectively $30 \%, 44 \%$ and $78 \%$. We see that the proposed protocols perform better than LEACH protocol.

\section{CONCLUSION AND FUTURE WORK}

The use of energy efficiently in sensor nodes is the most important issue in wireless sensor network in which the routing between the sensor nodes are considered as the most important one. In this paper, we proposed a new routing protocol in order to enlarge the life-time of sensor networks. This protocol developed from LEACH protocol by considering energy and distance of nodes in WSN in CHs election.

However, this protocol is only applied in the case of BS in the sensor area. But with BS is far from sensor area, we can not apply this protocol. In the future, we will study the energy distribution of node in the case BS is far from the sensor area to improve the lifetime of the whole network.

\section{REFERENCES}

[1] W. Heinzelman, A. Chandrakasan, and H. Balakrishnan, "Energy-efficient routing protocols for wireless microsensor networks," in Proc. $33^{\text {rd }}$ Hawaii Int. Conf. SystemSciences(HICSS), Maui, HI,Jan. 2000.

[2] Sarayoot Tanessakulwattana, Chotipat Pornavalai, Goutam Chakraborty. "Adaptive Multi-hop Routing for Wireless Sensor Networks", 10th International Joint Conference on Computer Science and Software Engineering (JCSSE), pp 105-110, 2013.

[3] Handy M, Haase M, Timmermann D., "Low Energy Adaptive Clustering Hierarchy with Deterministic Cluster-Head Selection," In: Proc. Of the 4th IEEE Conf. on Mobile and Wireless Communications Networks, pp. 368-372, 2002.

[4] Tauseef Shah, Nadeem Javaid, Talha Naeem Qureshi, "Energy Efficient Sleep Awake Aware (EESAA) Intelligent Sensor Network Routing Protocol”, 15th IEEE International Multi Topic Conference (INMIC'12), Pakistan, 2012.

\section{Authors}

TRAN CONG HUNG was born in Vietnam in 1961

He received the B.E in electronic and Telecommunication engineering with first class honors from HOCHIMINH University of technology in Vietnam, 1987.He received the B.E in informatics and computer engineering from HOCHIMINH University of technology in Vietnam, 1995.He received the master of engineering degree in telecommunications engineering course from postgraduate department Hanoi University of technology in Vietnam, 1998.He received Ph.D at Hanoi University of technology in Vietnam, 2004. His main research areas are B - ISDN performance parameters and

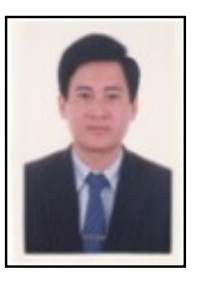
measuring methods, QoS in high speed networks, MPLS. He is, currently, Associate Professor PhD. of Faculty of Information Technology II, Posts and Telecoms Institute of Technology in HOCHIMINH, Vietnam.

\section{Nguyen Hong Quan was born in Vietnam in 1989}

Obtained BE in Electronic and Telecommunication from University of Sciences, Vietnam, 2011.Will receive Master degree in University of Sciences, 2014 major in Electronic and Telecommunication.He is, currently, Engineer of TMA Solution.

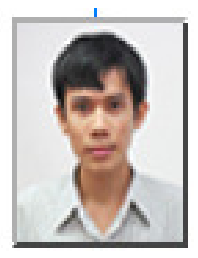

